\title{
Tinips
} PHARMACEUTICAL SCIENCES

\section{BIOANALYTICAL METHOD DEVELOPMENT AND VALIDATION OF TENELIGLIPTIN USING RP-HPLC IN RABBIT PLASMA \\ M.Anusha ${ }^{1 *}$ and Nallakumar Ponnu Swamy ${ }^{2}$}

${ }^{*}, 1$ Department of Pharmaceutical Analysis, Sri Venkateshwara College of Pharmacy and Research Centre, Madhapur, Hyderabad - 500081, India.

${ }^{2}$ Assistant Professor, Department of Pharmaceutical Analysis, Sri Venkateshwara College of Pharmacy and Research Centre, Madhapur, Hyderabad - 500081, India.

\begin{abstract}
:
A simple, highly sensitive, precise and accurate high-performance liquid chromatographic (HPLC) method was developed and validated for the determination of teneligliptin in rabbit plasma samples. The chromatographic

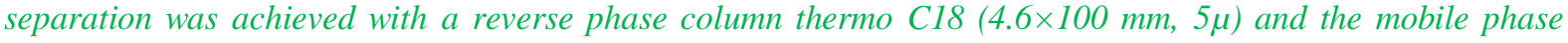
consisted of methanol and $5 \mathrm{~mm}$ potassium phosphate buffer $(60: 40 \mathrm{v} / \mathrm{v})$ at a flow rate of $1 \mathrm{~mL} / \mathrm{min}$. Sitagliptin was used as an internal standard. The retention time of Teneligliptin and sitagliptin were found to be 3.9 and 2.2 min respectively. The calibration curve was linear $(r 2>$ or $=0.99)$ ranging from 7.20 to $470 \mathrm{ng} / \mathrm{ml}$ and the lower limit of quantification was $7.20 \mathrm{ng} / \mathrm{mL}$. Interday precision were lower than $5 \%(\mathrm{CV})$ and accuracy ranged from 90 to $110 \%$ in terms of percent accuracy. Mean extraction recovery was found to be above $82 \%$. The method was successfully developed and validated in rabbit plasma for excellent selectivity, accuracy, precision, recovery and stability.
\end{abstract}

Keywords: HPLC; Teneligliptin; internal standard, Rabbit plasma; Validation

Corresponding author:

Nallakumar Ponnu Swamy,

Assistant Professor,

Department of Pharmaceutical Analysis,

Sri Venkateshwara College of Pharmacy and Research Centre,

Madhapur, Hyderabad - 500081, India

QR code

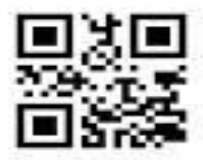

E-Mail: nallakumarponnuswamy@gmail.com

Mobile: +91964200862

Please cite this article in press as M.Anusha and N Ponnu Swamy, Bioanalytical Method Development and Validation of Teneligliptin UsingRP-HPLC in rabbit plasma, Indo Am. J. P. Sci, 2017; 4(08). 
INTRODUCTION:

Teneligliptin has the molecular formula $\mathrm{C}_{22} \mathrm{H}_{30} \mathrm{~N}_{6} \mathrm{OS}$ and chemical name $\{(2 \mathrm{~S}, 4 \mathrm{~S})-4$-[4-(3Methyl-1-phenyl-1H-pyrazol-5-yl)-1-piperazinyl]2- pyrrolidinyl \}(1,3-thiazolidin-3-yl)methanone with a molecular mass of $426.583 \mathrm{~g} / \mathrm{mol}$. and absorption maxima around $244 \mathrm{~nm}$. Teneligliptin belong to class of Antidiabetic drugs known as dipeptidyl peptidase inhibitors or gliptins.Teneliglitpin got FDA approval in 19 mainly for the treatment of diabetic. The mode of action of the teneligliptin is the glucagon-like peptide-1 (GLP-1) is secreted from alimentary canal in response to meal that promotes insulin secretion from pancreas and regulates blood sugar post meal by controlling glucagon secretion [1-5]. Teneligliptin exhibits a hypoglycemic effect by controlling the degradation of GLP-1 by inhibiting dipeptidyl peptidase-4 (DPP-4) activity and thereby increasing blood concentration of active GLP-1.

\section{Pharmacokinetics:}

After oral administration of a single $20 \mathrm{mg}$ and 40 $\mathrm{mg}$ dose to healthy subjects, teneligliptin was rapidly absorbed, with peak plasma concentrations (mean $\mathrm{T}$ max) occurring at 1.8 hours and 1 hour post dose. Plasma AUC of teneligliptin increased in a dose-proportional manner. Following a single oral $20 \mathrm{mg}$ and $40 \mathrm{mg}$ dose to healthy volunteers, mean plasma AUC of teneligliptin was 2028.9 and $3705.1 \mathrm{ng} * \mathrm{hr} / \mathrm{ml}$, Cmax was 187.2 and 382.4 $\mathrm{ng} / \mathrm{ml}$, and apparent terminal halflife (t1/2 ) was 24.2 and 20.8 hours. Plasma AUC of teneligliptin increased following $20 \mathrm{mg}$ doses at steady-state compared to the first dose. Coadministration with food reduces the Cmax by $20 \%$, increases the Tmax from 1.1 to 2.6 hours but does not affect the AUC of teneligliptin as compared to that in the fasting state. The plasma protein binding rate is $77.6-$ $82.2 \%$. Following a $20 \mathrm{mg}$ single oral dose of [14C] teneligliptin, 5 metabolites M1, M2, M3, M4 and M5 were observed. In vitro studies indicated that CYP3A4 and flavin-containing monooxygenase (FMO1 and FMO3) are involved in the metabolism of teneligliptin. Teneligliptin does not inhibit CYP1A2, CYP2A6, CYP2B6, CYP2C8, CYP2C8/9, CYP2C19, CYP2E1, is a weak inhibitor of CYP2D6, CYP3A4, and FMO (IC50 value : 489.4, 197.5 and $467.2 \mu \mathrm{mol} / \mathrm{l}$ ) and does not induce CYP3A4 and CYP1A2. Following a $20 \mathrm{mg}$ single oral dose of [14C] teneligliptin, $45.4 \%$ of administered radioactivity was excreted in urine and $46.5 \%$ in faeces till 216 hours after dose. The cumulative urinary excretion rates for upto 120 hours for un-metabolized, M1, M2, and M3 were $14.8 \%, 17.7 \%, 1.4 \%$ and $1.9 \%$ respectively while the cumulative faecal excretion rates for un-metabolized, M1, M3, M4 and M5 were $26.1 \%, 4.0 \%, 1.6 \%, 0.3 \%$ and $1.3 \%$ respectively. The single administration of teneligliptin at $20 \mathrm{mg}$ in patients with renal impairment revealed no remarkable changes in Cmax and $t 1 / 2$ corresponding to the level of renal impairment. Compared with healthy adult subjects,

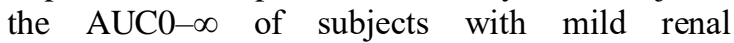
impairment $(50 \leq$ creatinine clearance $[\mathrm{Ccr}] \leq 80$ $\mathrm{mL} /$ minute), moderate renal impairment $(30 \leq \mathrm{Ccr}$ $<50 \mathrm{~mL} /$ minute), and severe renal impairment (Ccr $<30 \mathrm{~mL} /$ minute) was approximately 1.25 times, 1.68 times, and 1.49 times higher than that of healthy adult subjects, respectively. A single administration of teneligliptin $20 \mathrm{mg}$ in patients with hepatic impairment revealed that the Cmax of subjects with mild hepatic impairment (Child-Pugh classification: total score 5-6) and moderate hepatic impairment (Child-Pugh classification: total score 7-9) was approximately 1.25 times and 1.38 times that of healthy adult subjects, respectively.

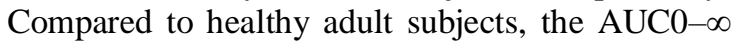
of subjects with mild and moderate hepatic impairments was approximately 1.46 times and 1.59 times higher than that of healthy adult subjects, respectively. There have been no previous clinical studies using teneligliptin in patients with severe hepatic impairment (Child-Pugh classification: total score was greater than 9).

\section{Adverse effects:}

The most common adverse reactions reported with teneligliptin are hypoglycemia and constipation. Other adverse reactions reported with teneligliptin are: Gastrointestinal Disorders: Intestinal obstruction, abdominal bloating, abdominal discomfort, nausea, abdominal pain, flatulence, stomatitis, gastric polyps, colon polyps, duodenal ulcer, reflux esophagitis, diarrhea, loss of appetite, increased amylase, lipase increased, acute pancreatitis [6-10]. Kidney and Urinary system: Proteinuria, urine ketone-positive. Skin and Subcutaneous Tissue Disorders: Eczema, rash, itching, allergic dermatitis. Investigations: Increase in AST, ALT, $\gamma$-GTP and ALP. Others: Increased $\mathrm{CPK}$, increased serum potassium, fatigue, allergic rhinitis, elevation of serum uric acid.

\section{Over Dosage:}

In the event of an overdose, it is reasonable to employ the usual supportive measures, e.g., remove unabsorbed material from the gastrointestinal tract, employ clinical monitoring (including obtaining an electrocardiogram), and institute supportive therapy as dictated by the patient's clinical status.

\section{Warnings and Precautions:}

Teneligliptin should be administered carefully in the following: Patients with advanced liver failure (safety has not been established),Patients with congestive heart failure (NYHA category III-IV) (safety has not been established), Patients with 
pituitary insufficiency or adrenal insufficiency, poor nutritional state, starvation, an irregular dietary intake, or debilitating condition, intense muscle movement or excessive alcohol intake (may cause low blood sugar), Patients with history of abdominal surgery or with a history of bowel obstruction (may cause bowel obstruction), Patients with arrhythmia, severe bradycardia or its history, patients with heart disease such as congestive heart failure or patients with low serum potassium, congenital prolonged QT syndrome, history of Torsades de pointes or patients using antiarrhythmic drugs (may cause QT prolongation), Patients using an insulin secretagogue (e.g., sulfonylurea) (risk of severe hypoglycaemia).

Drug name: Teneligliptin

\section{Chemical Formula: $\mathrm{C}_{22} \mathrm{H}_{30} \mathrm{~N}_{6} \mathrm{OS}$}

Chemical Structure: $\{(2 \mathrm{~S}, 4 \mathrm{~S})-4-[4-(3-M e t h y l-1-$ phenyl-1H-pyrazol-5-yl)-1-piperazinyl]-2pyrrolidinyl $\}$ (1,3-thiazolidin-3-yl)methanone.

Molecular Weight: $426.22 \mathrm{~g} / \mathrm{mol}$

Solubility: in water, DMSO,methanol

PKa: 8.7

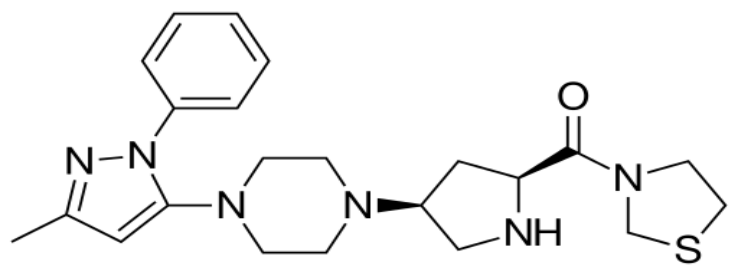

Fig 1 : chemical structure of Teneliglitpin

Drug name: Sitagliptin

Chemical Formula: $\mathrm{C}_{16} \mathrm{H}_{15} \mathrm{~F}_{6} \mathrm{~N}_{5} \mathrm{O}$

Chemical Structure: (3R)-3-amino-13(trifluoromethyl)-5H,6H,7H,8H$[1,2,4]$ triazolo[4,3-a]pyrazin-7-yl]-4-(2,4,5trifluorophenyl)butan-1-one

Molecular Weight: $407.32 \mathrm{~g} / \mathrm{mol}$

Solubility: in water, $179.2 \mathrm{mg} / \mathrm{L}$ at $25^{\circ} \mathrm{C}$

LogP : 1.5

PKa : 8.78<smiles>N[C@@H](CC(=O)N1CCn2c(nnc2C(F)(F)F)C1)Cc1cc(F)c(F)cc1F</smiles>

Fig 2: chemical structure of sitagliptin

\section{Literature survey}

Literature survey has revealed that there is only single method is reported for the determination of Teneligliptin in human plasma by LCMS/MS with the chromatographic conditions of run time 1.50 min run time, separation was achieved on a Hypersil Gold C18 column using a mobile phase composed of $0.1 \%$ formic acid in Milli-Q water $/ 0.1 \%$ formic acid in acetonitrile in gradient elution mode. The quantification of teneligliptin was performed in a positive electro spray ionization mode and multiple reaction monitoring (MRM) Run time:10mins,Hypersil gold column,mobile phase $: 0.1 \%$ formic acid in Milli-Q water/0.1\% formic acid in acetonitrile. Methods reported in the another is that the estimation of Teneligliptin in bulk and pharmaceutical dosage forms by RPHPLC with the chromatographic conditions of and another Method reported in the literature for the estimation of Teneligliptin in bulk and pharmaceutical dosage forms by UV spectroscopy and HPTLC of conditions are solvent system consisted of toluene: chloroform: ethanol: diethylamine in the proportion of $4: 4: 1: 1, \mathrm{v} / \mathrm{v} / \mathrm{v} / \mathrm{v}$. Methods reported in the another is that the estimation of Teneligliptin in bulk and degradation forms by RP-HPLC with the chromatographic conditions for Separation was achieved on a Shisedo C18column, $5 \mu \mathrm{m}, 250 \mathrm{~mm} \times 4.6 \mathrm{~mm}$ i.e. column using a mobile phase consisting of Acetonitrile:Methanol: Water (30:40: $30 \% \mathrm{v} / \mathrm{v} / \mathrm{v}$ ) at a flow rate of $1.0 \mathrm{ml} / \mathrm{min}$ and $\mathrm{UV}$ detection at 246nm. Estimation of Anti-Diabetic Teneligliptin Hydrobromide Hydrate by Rp-Hplc and Derivative Spectroscopic Method with the Isocratic elution at the flow rate of $1.0 \mathrm{ml} / \mathrm{min}$ was employed on a Kromasil 100-5-C8 column at ambient temperature. The mobile phase consisted of Methanol: $0.025 \mathrm{M}$ phosphate buffer $\mathrm{pH}$ adjusted to 3 with o-phosphoric acid (60:40 v/v) and the detection wavelength was at $254 \mathrm{~nm}$ [11]. 


\section{MATERIALS AND METHODS:}

\section{Chemicals and reagents}

Teneligliptin (Figure 1) of the highest quality has been purchased from Microlabs and sitagliptin (Internal Standard) (Figure 2) was donated by MSD. HPLC grade Methanol 99.8\% was obtained from Merck chemicals; Hplc grade water and ethylacetate were of analytical reagent grade supplied by Merck Chemicals.

\section{Instrumentation}

WATERS HPLC, Model: Aglient 2695, Photo diode array detector (PDA), with an automated sample injector. The output signal was monitored and integrated using Empower 2 software.

\section{Chromatographic conditions}

Chromatographic separations were achieved by using Thermo C18 column $(250 \times 4.6 \mathrm{~mm}, 5 \mu \mathrm{m})$. The mobile phase consisted of methanol and $5 \mathrm{~mm}$ potassium hydrogen phosphate buffer in the ratio of 60:40 v/v. runtime -7 miuntes and flow rate of $1 \mathrm{~mL} / \mathrm{min}$ and the injection volume was $20 \mu \mathrm{l}$. the column oven was kept at $25^{\circ} \mathrm{C}$ throughout the analysis. The eluent was detected by PDA detector.

\section{Preparation of stock and standard solutions}

Teneligliptin was weighed and dissolved in methanol and concentrations made to $1000 \mu \mathrm{g} / \mathrm{ml}$ and futher samples are prepared of eight different standard concentrations of 7.20, 20.24, 47.060, $117.660,213.940,305.620$, and $470.00 \mathrm{ng} / \mathrm{ml}$. The QC samples are prepared at four different levels are HQC, MQC, LQC and LLOQ. All the standard concentration and QC samples are prepared by using methanol and water (80:20) as diluents.

\section{Extraction method}

Extraction of teneligliptin from rabbit plasma sample was carried out by using liquid-liquid extraction. Each standard concentration sample and QC sample are individually taken and pipette out $0.25 \mathrm{ml}$ of sample and $0.1 \mathrm{ml}$ of internal standard into centrifuge tubes and add plasma $\&$ to the tube add $3 \mathrm{ml}$ of ethylacetate and centrifuge for 15 minutes (2500rpm).then evaporate the contents and reconstitute the tube with mobile phase and filled in the HPLC vials and run. The representative chromatograms were shown in Figure 2.

\section{Bioanalytical method validation}

Preparation of calibration curve: the linearity of the method was evaluated by a calibration curve in the range of $7.20-490 \mathrm{ng} / \mathrm{mL}$ of teneligliptin. The calibration curve was achieved by plotting the peak area ratios of teneligliptin versus the concentration of teneligliptin by least-squares linear regression analysis. The calibration curve requires a correlation coefficient (R2) of >0.999. The acceptance criteria for each back-calculated standard concentration should be within $15 \%$ of the nominal concentration, except it should not exceed $20 \%$ for the LLOQ.(table-1)

\section{Accuracy and precision:}

Intra-day accuracy and precision were determined by duplicate analysis of six sets of samples spiked with four different concentrations of teneligliptin at low, medium, high quality control samples $(18.000,211.680,376.000 \mathrm{ng} / \mathrm{mL})$ including LLOQ $(7.200 \mathrm{ng} / \mathrm{mL})$ within a day or on 6 consecutive days. For acceptance criteria for intra and inter-day precision, accuracy should be within $85-115 \%$ of the nominal concentration and coefficient of variation $(\% \mathrm{CV})$ values should be $<15 \%$ over the calibration range, except at the LLOQ, where accuracy should be between $80-120 \%$ and $\% \mathrm{CV}$ should not be more than $20 \%$.(table-2)

\section{Selectivity:}

The selectivity of the assay methodology was established using a minimum of six independent sources of the same matrix. There were no interferences from the endogenous material at the retention time for both teneligliptin and internal standard (sitagliptin). The representative chromatogram is shown in Figure 3.

\section{Recovery:}

Recovery of teneligliptin was evaluated by comparing the mean peak areas of three extracted low, medium and high quality control samples to mean peak areas of three neat reference solutions (un-extracted). Recovery of internal standard was evaluated at a concentration of $210 \mathrm{ng} / \mathrm{mL}$ and corresponding mean peak area of the extracted samples compared to the mean peak areas of neat reference solutions. Recovery of the analyte need not be $100 \%$, but the extent of recovery for analyte (teneligliptin) and internal standard (sitagliptin) should be consistent and reproducible.

\section{Stability:}

In order to find out the stability of teneligliptin in rabbit plasma, bench top stability, freeze thaw stability, and long term stability studies were carried out by using six replicates of the low and high plasma quality control samples. For the bench top stability, frozen plasma samples were kept at room temperature for $24 \mathrm{hr}$ before sample preparation. Freeze-thaw stability of the samples was obtained over three freeze-thaw cycles, by thawing at room temperature for 2-3 $\mathrm{hr}$ and refrozen for $12-24 \mathrm{hr}$ for each cycle. Freshly prepared solutions are taken and analysed after 24hours and Long term stability of teneligliptin in rabbit plasma was tested after 3 days. For the acceptance criteria of stability, the deviation compared to the freshly prepared standard should be within $\pm 15 \%$ of the nominal concentration. 


\section{Matrix effect:}

The matrix effect was performed in 6 different lots of rabbit plasma by taking aqueous samples and spiked plasma samples at HQC and LQC level (6replicates of each).Matrix suppression or enhancement was calculated as follows: $100 \times$ mean peak area of post extracted sample/mean peak area of neat standard solution. The acceptance criteria for matrix effect implied that the \% CV should be less than $15 \%$ of matrices tested and at least $80 \%$ of matrices should meet the above criteria. the results obtained were displayed in Table 4.

Table 1: Calibration table

\begin{tabular}{|c|c|c|c|}
\hline Concentration(ng/ml) & $\begin{array}{c}\text { Area } \\
\text { Ratio }\end{array}$ & back.cal.con & $\%$ concentration \\
\hline 7.080 & 0.018 & 7.377 & 104.2 \\
\hline 20.240 & 0.050 & 20.497 & 101.3 \\
\hline 47.060 & 0.117 & 47.277 & 100.5 \\
\hline 117.660 & 0.293 & 117.883 & 100.2 \\
\hline 213.940 & 0.529 & 212.514 & 99.3 \\
\hline 305.620 & 0.760 & 305.080 & 99.8 \\
\hline 399.500 & 0.997 & 399.930 & 100.1 \\
\hline 470.000 & 1.173 & 470.541 & 100.1 \\
\hline
\end{tabular}

Table 2: precision and accuracy $(n=6)$.

\begin{tabular}{|c|c|c|c|c|}
\hline \multirow{2}{*}{ QC Sample } & \multicolumn{4}{|c|}{ Teneligliptin } \\
\cline { 2 - 5 } & LLOQ & LQC & MQC & HQC \\
\hline $\begin{array}{c}\text { Theoretical } \\
\text { concentration } \\
(\mathrm{ng} / \mathrm{mL})\end{array}$ & 7.200 & 18.000 & 211.680 & 376.000 \\
\hline \multicolumn{5}{|c|}{ precision and accuracy } \\
\hline $\begin{array}{c}\text { Mean estimated } \\
\text { concentration } \\
\text { (ng/mL) } \pm \text { SD }\end{array}$ & $7.107 \pm 0.04$ & $17.210 \pm 0.05$ & $199.478 \pm 0.07$ & $354.871 \pm 0.40$ \\
\hline Precision (CV,\%) & 0.17 & 0.2 & 0.150 & 0.644 \\
\hline Accuracy (\%) & 98.7 & 95.63 & 95.10 & 94.36 \\
\hline
\end{tabular}

Table 3: Extraction Recovery $(n=6)$.

\begin{tabular}{|c|c|c|c|}
\hline \multirow{2}{*}{ teneligliptin } & LQC \% & MQC\% & HQC\% \\
\hline \multirow{2}{*}{$\begin{array}{c}\text { Extraction } \\
\text { recovery } \\
\text { of six } \\
\text { different } \\
\text { aliquots of } \\
\text { rabbit } \\
\text { plasma }\end{array}$} & 101.4745 & 101.521 & 100.00 \\
\cline { 2 - 4 } & 103.0403 & 102.7362 & 101.3169 \\
\cline { 2 - 4 } & 103.0584 & 101.952 & 107.0072 \\
\cline { 2 - 4 } & 101.3758 & 103.6097 & 103.8113 \\
\cline { 2 - 4 } & 89.14724 & 103.3616 & 114.2069 \\
\hline \multirow{2}{*}{$\begin{array}{c}\text { Mean of } \\
\text { extraction } \\
\text { recovery }\end{array}$} & 99.20366 & 102.6733 & 105.6888 \\
\hline \multicolumn{2}{|c|}{ Sitagliptin (IS) } & \multicolumn{2}{|c|}{99.05} \\
\hline
\end{tabular}


Table 4: Matrix effect $(n=6)$.

\begin{tabular}{|c|c|c|}
\hline \multirow{4}{*}{ Teneligliptin } & Extracted area & Unextracted area \\
\hline \multirow{4}{*}{ HQC } & 1007715 & 935722 \\
\cline { 2 - 3 } & 1007623 & 934799 \\
\cline { 2 - 3 } & 1006654 & 993570 \\
\cline { 2 - 3 } & 1005683 & 939827 \\
\cline { 2 - 3 } & 1004378 & 967504 \\
\cline { 2 - 3 } & 1073892 & 940304 \\
\hline Average area & 1017657.5 & 951954.3 \\
\hline \multicolumn{2}{|c|}{ M.E at HQC $(\%)$} \\
\hline
\end{tabular}

\begin{tabular}{|c|c|c|}
\hline Teneligliptin & Extracted area & Unextracted area \\
\hline \multirow{4}{*}{ LQC } & 48241 & 47540 \\
\cline { 2 - 3 } & 47241 & 48639 \\
\cline { 2 - 3 } & 48567 & 47134 \\
\cline { 2 - 3 } & 49872 & 48392 \\
\cline { 2 - 3 } & 47895 & 47245 \\
\cline { 2 - 3 } & 42098 & 47223 \\
\hline Average area & 47319.0 & 47695.5 \\
\hline M.E at LQC $(\%)$ & 99.21061735 \\
\hline
\end{tabular}

Table 5: Stability details of teneligliptin in rabbit plasma sample $(n=6)$

\begin{tabular}{|c|c|c|}
\hline Sample name & Mean concentration \pm SD & \%cv \\
\hline \multicolumn{3}{|c|}{ Freeze thaw stability } \\
\hline LQC & $17.16 \pm 0.05$ & O.29 \\
\hline HQC & $354 \pm 0.46$ & 0.12 \\
\hline \multicolumn{3}{|c|}{ Short term stability } \\
\hline LQC & $17.14 \pm 0.11$ & 0.64 \\
\hline HQC & $356 \pm 0.12$ & 0.31 \\
\hline LQC & Long term stability \\
\hline HQC & $17.14 \pm 0.12$ & 0.70 \\
\hline
\end{tabular}

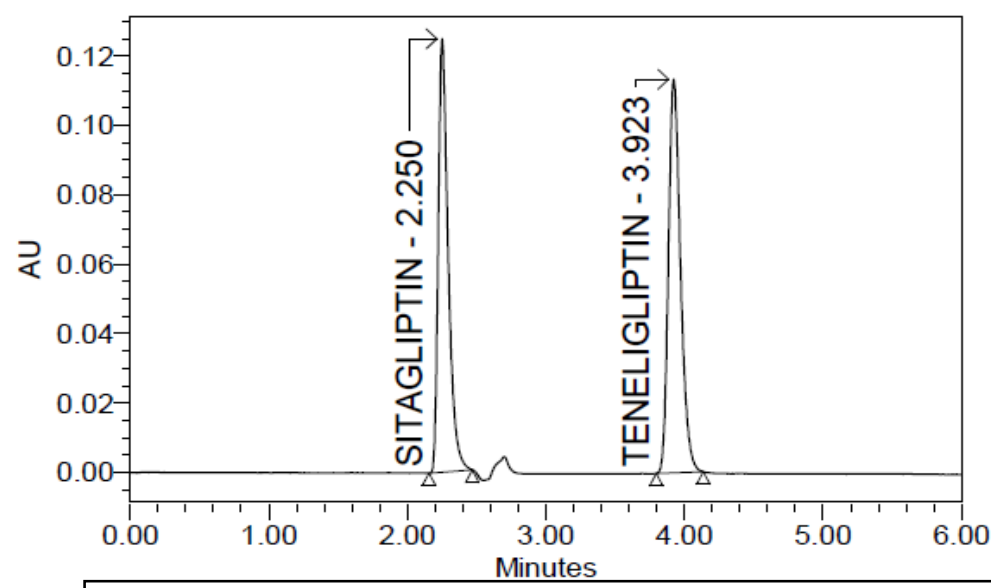

Fig 3 : Representative chromatogram of sitagliptin and Teneligliptin in rabbit plasma. 


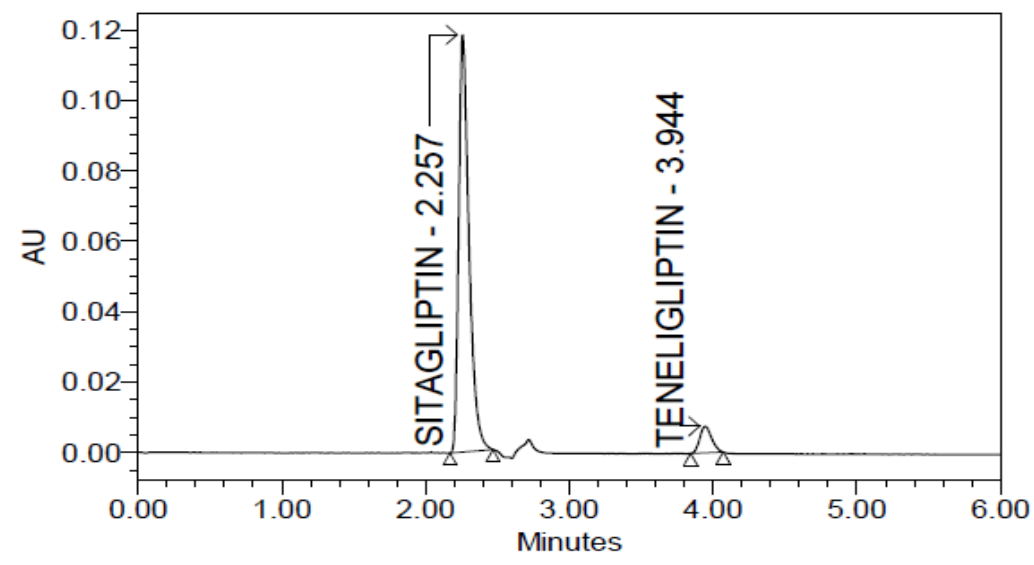

Fig 4: Representative chromatogram of Teneligliptin and sitagliptin at lower limit of quantification in rabbit plasma.

\section{RESULTS:}

\section{Selectivity and optimization of chromatographic conditions}

Plasma matrices were obtained from six different sources and assayed to evaluate the selectivity of the method and the detection of interference. Teneligliptin and sitagliptin (internal standard) were well separated from the co-extracted material under the described chromatographic conditions at retention times of 3.9 and $2.2 \mathrm{~min}$ respectively. No endogenous peak from plasma was found to interfere with the elution of either the drug or the internal standard. The LLOQ which could be measured with acceptable accuracy and precision for the analyte $7.20 \mathrm{ng} / \mathrm{mL}$ was established (Figure 4). It indicates that the proposed method is highly selective and specific.

\section{Calibration curve}

Linear detector response for the peak-area ratios of the teneligliptin was observed in the concentration range between $7.20-470 \mathrm{ng} / \mathrm{mL}$ with a mean correlation coefficient of 0.999 . the reason for choosing a wide calibration range for teneligliptin is to analyze samples of higher and lower dose concentration for route of administration like oral route where the Cmax concentration, best it for the calibration curve could be achieved with the linear equation $\mathrm{Y}=\mathrm{mx}+\mathrm{c}$. where $\mathrm{Y}$ was the peak area ratio of the analyte and $\mathrm{x}$ was the concentration of the analyte. the results were given in the Table 1.

\section{Accuracy and precision}

The intra-day accuracy and precision ranged between 94.36-98-7\%, and 0.17-0.64\%, respectively. The accuracy and precision at the LLOQ and at LQC, MQC, HQC control samples of teneligliptin in plasma were within acceptable limits $(\mathrm{N}=6)$. The results of the method validation studies presented in Table2.

\section{Matrix effect}

The sample solutions are prepared at two QC levels that is HQC and LQC one is of aqueous format and other is of spiked plasma. matrix effect is calculated that is average area observed at QC level in matrix sample by average area observed at QC level in aqueous sample * 100.the matrix effect studies presented in table 4 .

\section{Recovery}

The recovery of teneligliptin in plasma was calculated at three QC levels. the response (extracted) compared to that of unextracted samples of the reference solution. The percentage recovery of teneligliptin and sitagliptin (internal standard) were found to be above $85 \%$. Results are displayed in the Table 3.

\section{Stability}

Stock solutions of teneligliptin $(1 \mathrm{mg} / \mathrm{mL})$ and internal standard $(1 \mathrm{mg} / \mathrm{mL})$ were separately prepared and diluted at two levels LQC and HQC. The stability experiments were aimed at testing all possible conditions that the samples might experience after collecting and prior the analysis. All stability results were summarized in Table 5 . the results of three freeze- thaw cycles and when the spiked samples were kept at room temperature indicated that teneligliptin was stable in rabbit plasma under these conditions. Analyte spiked QC samples were stable .

\section{Application of the method}

The method had been successfully employed for the quantitative estimation of teneligliptin in rabbit plasma samples. This Bioanalytical method has selectivity, sensitivity and reproducible. 


\section{DISCUSSION:}

\section{RP-HPLC optimization}

The RP-HPLC method for the detection of Teneligliptin in rabbit plasma was investigated. Teneligliptin was dissolved in methanol to obtain the primary stock solution followed by subsequent dilution in methanol: water (80:20) and was directly introduced into the HPLC and various conditions are maintained and checked out for the response.

During the early stages of method development, attempts were made to optimize the response in a perfect manner. And. When standard working stock solutions of the analyte were directly injected into the HPLC system, it was observed that the response for the analyte was linear over the mentioned calibrated range. The linearity was found to be best it for the calibration range when the compound was dissolved in methanol: water (80:20) for preparing the working stock solution. the buffer $\mathrm{pH}$ at 4 helped in increasing the linear response by influencing the column properties like retention time, peak shape and peak response with relative variation of $15 \%$ for the analyte. It is accepted as a fact that a non linear range would not be adequate to the pharmacokinetic study. But by controlling the variable parameters like solubility, $\mathrm{pH}$, column, temperature, ionization, less injection volume, obtaining a huge linear range with less possible relative error is possible. As per this method, flow rate and injection volume was set at $1 \mathrm{~mL} / \mathrm{min}$ and $10 \mu \mathrm{L}$ injection volume. Generally split ratio tends to change with changes in back pressure and expensive in terms of high solvent consumption. But, in this method, no split employed and that possibly decreases solvent consumption, improves reproducibility, increase column life and simultaneously declines chemical noise. It is also important for selection of column, column dimensions $(250 \mathrm{~mm} \times 4.6 \mathrm{~mm})$, indicating fast elution with shorter run time. Hence, this method is validated for the linearity range from $7.20-470$ $\mathrm{ng} / \mathrm{mL}$. During an early phase of method development attempts were made to choose the right column, since the columns like waters $\mathrm{C} 18$, Supelco C18, Zodiac C18 showed that the analyte eluted within the void volume with bad peak shape and poor area response, but as we tried to improve retention time through $\mathrm{C} 18$ columns, even though the area response and peak shape was improved considerably. The best C18 column, chosen based on separation, reproducibility, and response. Taking into account the non polarity nature of the teneligliptin, liquid liquid extraction had been proven to be an effective technique in the published methods. However, liquid liquid extraction was found to be LLE provides efficient removal of analyte with desired specificity/selectivity required for intended bioanalysis. Employing a methanol and ethylacetate for the extraction and constituted with mobile phase. Since, the reported bioanalytical methods for the estimation of teneligliptin by LC-MS/MS in human plasma. But, the present method is determination of Teneligliptin in rabbit biological samples by RPHPLC.In this proposed method, no interfering peaks were observed at the elution times of Teneligliptin and sitagliptin (IS). The method also had sufficient selectivity, specificity, precision and accuracy over the concentration range of 7.20-490 $\mathrm{ng} / \mathrm{mL}$. This method had a quantification limit of $7.20 \mathrm{ng} / \mathrm{mL}$, which was adequate enough to quantify the drug in rabbit plasma.

\section{CONCLUSION:}

Since merits of HPLC compare to other techniques are well recognized, a highly sensitive, specific, less cost compared to LCMS/MS and reproducible HPLC method is more valuable. In addition, along with method development, the method is also validated to quantify the concentration range of $7.20-470 \mathrm{ng} / \mathrm{mL}$ of Teneligliptin in rabbit plasma samples. The HPLC method presented here fulfils the criteria generally required for the Bioanalytical assays. This Bioanalytical method has sensitivity, selectivity and recovery above $85 \%$.

\section{ACKNOWLEDGEMENT}

Authors are thankful to Principal Dr. Bhagavan Raju Sri Venkateshwara College Of Pharmacy for support, encouragement and providing facilities to carry out the work. Also I am thankful to Osmania University, Hyderabad, who extended their support for project.

\section{REFERENCES:}

1.Guidance for industry, Bioanalytical method validation, U.S. Department of Health and Human Services Food and Drug Administration Centre for Drug Evaluation and Research, Centre for Veterinary Medicine, 2013, P 1-23.

2.Guideline on Bioanalytical method validation, European medicines agency, 2011, P 1-23.

3.Chunduri et al. World Journal of Pharmacy and Pharmaceutical Sciences,Development And Validation Of Lc-ms/Ms Method For Quantification Of Teneligliptin In Human Plasma And Its Application To A Pharmacokinetic StudyVolume 5, Issue 5, 838-850 ,ISSN 2278 4357.

4.Vishnu C. Shinde et al Der Pharmacia Lettre, 2016, 8 (8):291-301Development and validation of UV spectrophotometric method and high performance thin layer chromatographic (HPTLC) method for estimation of Teneligliptin hydrobromide in pharmaceutical preparation, Scholars Research Library.

5.Sohan S. Chitlange et al. ISSN NO: 2231-6876 Vol 6, Issue 07, 2016. Estimation Of Anti-diabetic Teneligliptin Hydrobromide Hydrate By Rp-hplc 
And Derivative Spectroscopic Method Indo American Journal of Pharmaceutical Research.

6.Charde Et Al /2013 Bioanalytical Method Development And Validation International Journal Of Advances In Pharmaceutical Analysis IJAPA Vol. 3 Issue 4 (2013) 90-94.

7.https://pubchem.ncbi.nlm.nih.gov/compound/Ten eligliptin

8.http://www.Drugbank.ca/drugs/DB01261

description about sitagliptin. 9.http://www.centaurpharma.com/newwebsite/dow nloads/TEN20.pdf about pharmaceutical information about TEN20mg.

10. Extraction of Drug from the Biological Matrix: A Review, Applied Biological Engineering Principles and Practice, www.intechopen.com

11.Bioanalytical Method Development and Validation by HPLC: A Review, Pushpa Latha et al.: Journal of Medical and Pharmaceutical Innovation; 1 (6S) 2014; 1-9.

\section{Abbreviations:}

CV: Coefficient Variation; HPLC: High Performance Liquid Chromatography; IS: Internal Standard; LC: Liquid Chromatography; LC-MS/MS: Liquid Chromatography -Mass Spectrometry / Mass Spectrometry; LLOQ: Lower Limit of Quantitation; LQC: Low Quality Control; MQC: Medium Quality Control; HQC: High Quality Control; PK: Pharmacokinetics; QC: Quality Control; RP: Reverse Phase; TK: Toxicokinetic studies; BCS: Biopharmaceutics Classiication System; UV: Ultraviolet spectrophotometry; $C_{\text {max }}$ : the maximum plasma concentration of the drug; psi: Pounds per square inch. 